EPJ Web of Conferences 85, 01007 (2015)

DOI: $10.1051 /$ epjconf/ 20158501007

(C) Owned by the authors, published by EDP Sciences, 2015

\title{
TMD and spin asymmetries in SIDIS
}

\author{
Andrea Bressan 1,a \\ ${ }^{1}$ Department of Physics, University of Trieste and Trieste Section of INFN, Trieste, Italy
}

\begin{abstract}
A review of the most recent measurements of leading twist transverse momentum dependent distribution and fragmentation function accessed in the semi-inclusive deep inelastic scattering HERMES, COMPASS and JLab experiments is given.
\end{abstract}

\section{Introduction}

The study of transverse momentum and transverse spin dependent effects in semi-inclusive deep inelastic scattering (SIDIS) has grown significantly in the last 15 years both theoretically and experimentally, also based on the measurements provided both by COMPASS [1-3] and HERMES [4, 5] and more recently by the Hall A experiment at JLab [6, 7]. These experiments demonstraded the existence of interactions coming from spin-orbit correlations inside the nucleon and in the fragmentation process, while theorist have worked out the complete description of the nucleon at leading twist $[8,9]$ and proven deep factorisation theorems $[10,11]$ leading to universality (or pseudouniversality) of the newly introduced transverse momentum dependent parton distribution (TMD PDF) and fragmentation (TMD FF) functions. As we know, if the quarks are perfectly collinear with the parent hadron (or if we integrate on the quark transverse momentum $k_{\perp}$ ), three distribution functions, i.e. the number density $f_{1}^{q}(x)$, the helicity distribution $g_{1}^{q}(x)=f_{+}^{q}(x)-f_{-}^{q}(x)$ and the transversity distribution $h_{1}^{q}(x)=f_{\uparrow}^{q}(x)-f_{\downarrow}^{q}(x)$ exhaust the information on the internal dynamics of hadrons at twist-two (here, the index $q$ denotes the quark flavour, \pm are positive/negative helicities, with respect to the parent nucleon helicity and $\uparrow \downarrow$ are parallel/antiparallel quark spins with respect to the transversely polarised parent nucleon. If instead we admit a non negligible quark transverse momentum, the number of distribution functions considerably increases. Spin in fact couples naturally to the intrinsic transverse momentum of quarks, and the resulting correlations are expressed by various transverse momentum dependent distribution and fragmentation functions, that give rise to a large number of possible single-spin, double spin and azimuthal asymmetries. At the leading twist, there are eight TMD PDFs describing different spin $/ k_{\perp}$ correlations, such as the correlation of the quark transverse momentum $k_{\perp}$ and the nucleon transverse spin (the most famous Sivers function $f_{1 T}^{\perp, q}$ ) or the quark transverse spin with its transverse momentum, inside an unpolarised nucleon (the Boer-Mulders

\footnotetext{
a e-mail: Andrea.Bressan @ cern.ch
}

function $h_{1}^{\perp, q}$ ). All the eight leading twist TMD PDFs are presented in fig. 1. Analogous correlations also occurs for fragmentation functions that describe the hadronisation of the struck quark into hadrons. Also here the quark spin couples naturally with the transverse momentum of the hadron generated in the hadronisation process, as is the case f.i. for the correlation between the quark transverse spin and the transverse momentum of the fragmenting hadron (the Collins fragmentation function $H_{1}^{\perp, q \rightarrow h}$ ). Analogously to the eight twist-two TMD PDFs also eight leading twist TMD FF can exist, denoted by capital letters, we have D (unpolarised), G (longitudinally polarised), H (transversely polarised) fragmentation functions. Thus, $D_{1}$ is the $p_{\perp}$ dependent extension of the usual leading-twist unpolarised fragmentation function, $G_{1}$ the fragmentation function of longitudinally polarised quarks, $H_{1}$ the fragmentation function of transversely polarised quarks.

In the SIDIS process $\ell(l)+N(P) \rightarrow \ell\left(l^{\prime}\right)+h\left(p_{h}\right)+X$, the convolution of TMD PDFs and FFs give rise to different modulations as a function of the azimuthal hadron angle $\phi_{h}$ and quark transverse spin $\phi_{S}$ [9]. These modulations are orthogonal among them and allow to separate the contributions of the different convolutions of TMD PDF and FF entering the cross section. Nevertheless one of these asymmetries always contains at least a convolutions of PDF and FF; to further separate the two terms one needs to combine the analysis of SIDIS experiments with those of $e^{+} e^{-}$colliders experiments where the modulations depend on convolutions of FF only. Doing global fits of SIDIS and $e^{+} e^{-}$ experiments further requires to evolve the TMD PDFs and FFs to the measured experimental points, with the evolution kernel of TMDs still being investigated with different approaches. The content in term of TMD PDFs and FFs of the different azimuthal asymmetries/modulations that can be accessed in unpolarized or transversely polarized SIDIS is given in table 1; the superscript of the asymmetry indicates the corresponding modulation, the first and the second subscripts the respective ('U'-unpolarized, 'L'longitudinal and ' $\mathrm{T}$ '-transverse) polarization of beam or target. As one can see, there are five Single-Spin Asymme- 


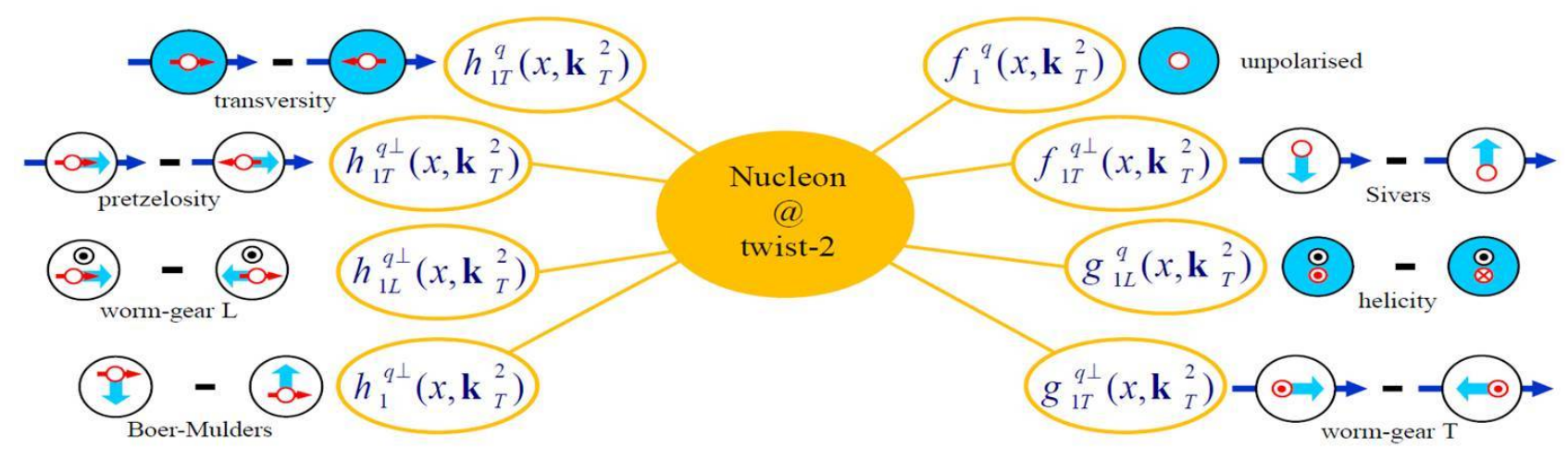

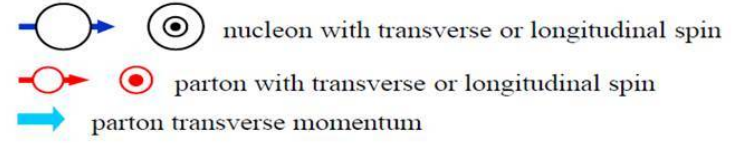

Proton goes out of the screen. Photon goes into the screen

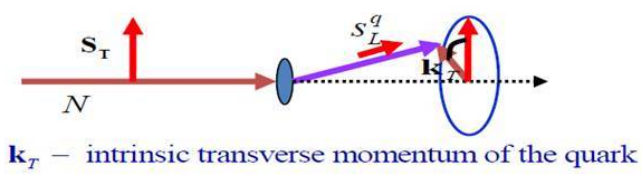

Figure 1. Nucleon description at leading twist when $k_{\perp}$ is considered

tries (SSA), which depend only on the transvere target spin $S_{T}$ and three Double-Spin Asymmetries (DSA), both $S_{T}$ and $P_{B}$ (beam polarization) dependent. In the QCD parton model approach four of the eight TSAs have Leading Order (LO) interpretation. The remaining four asymmetries are higher-twist effects, though they can be interpreted as Cahn kinematic corrections to twist-two spin effects on the transversely polarised nucleon.

SIDIS events are usually identified using standard cuts; some differences for different channels and experiments are due to the the beam energy and thus to the kinematic domain. The DIS events are selected requiring the photon's virtuality $Q^{2}$ to be larger than $1(\mathrm{GeV} / c)^{2}$, the fractional energy $y$ transferred from the beam lepton to the virtual photon to be larger than 0.1 (to remove events affected by poor energy resolution) and smaller than 0.9 (to limit the size of radiative corrections). A minimum value of invariant mass of the final hadronic state $W>2 \mathrm{GeV} / c$ is also needed to exclude the resonance region. Typically, values of $W>2,3.3$ and $5 \mathrm{GeV} / c^{2}$ are required in the data analyses of the JLab, HERMES and COMPASS experiments respectively.

The kinematic coverage in $x$ and $Q^{2}$ for the selected events strongly depends on the lepton's beam energy. Figure 2 shows the regions of the $\left(x, Q^{2}\right)$ plane kinematically accessible with lepton beams of $160,27.5$ and $6 \mathrm{GeV}$ energies, corresponding to the COMPASS, HERMES and JLab experiments respectively. In the COMPASS experiment the $x$ range is between 0.004 and 0.3 , where the upper limit is given by the low luminosity; for the HERMES experiment $0.02<x<0.4$, while the JLab experiments can presently measure with high precision at $x>0.1$, in the valence region. The average $Q^{2}$ values are also different and there is a strong $x-Q^{2}$ correlation. At $x \sim 0.1$ the mean $Q^{2}$ value is $6.4(\mathrm{GeV} / c)^{2}$ at COMPASS and about $2.5(\mathrm{GeV} / c)^{2}$ at HERMES. In the overlap region the ratio of the $Q^{2}$ mean values measured in the two experiments goes from 2 to 3 with increasing $x$, in spite of the similar mean values when integrating over the whole $x$ range. The differences in the covered kinematic regions make the experiments complementary, and, all together, they guarantee a good coverage of the phase space. The phase space is in any case limited when compared with the measurements of unpolarised PDFs performed at HERA in the nineties, with an unpolarised lepton-proton collider.

In addition to the requirements on the inclusive DIS variables cuts on the energy of hadrons are also applied, depending on the experiment and on the physics channel under consideration. In the single hadron analyses, the relative energy $z$ of each hadron has to be between 0.2 and 0.8 . The upper limit, usually not required by COMPASS, is chosen to reject exclusively produced hadrons. The lower limit is used to select hadrons from the current fragmentation region.

\section{Accessing transversity}

The main properties of the the transversity distribution $h_{1}$ are:

(i) it is chirally-odd and therefore does not appear in the handbag diagram of inclusive DIS, which cannot flip the chirality; in order to measure $h_{1}$, the chirality must be flipped twice, so one always needs two hadrons, both in the initial state, or one in the initial state and one in the final state, and at least one of them must be transversely polarised;

(ii) there is no gluon transversity distribution: this would imply a helicity-flip gluon-nucleon amplitude, which does not exist since gluons have helicity 1 and the nucleon cannot undergo an helicity change of two units;

(iii) the DGLAP equations for $h_{1}$ have been worked out up to next-to-leading order. Since there is no gluon 
Table 1. TMD PDF and FF content of azimuthal modulations/asymmetries accessible in unpolarised or transversely polarised SIDIS

\begin{tabular}{ll}
\hline$A_{U U}^{\cos \phi_{h}} \propto \frac{1}{Q}\left(f_{1}^{q} \otimes D_{1}^{q \rightarrow h}-h_{1}^{\perp, q} \otimes H_{1}^{\perp, q \rightarrow h}+\cdots\right)$ & $A_{L T}^{\cos \left(\phi_{h}-\phi_{S}\right)} \propto g_{1 T}^{q} \otimes D_{1}^{q \rightarrow h}+\cdots$ \\
$A_{U U}^{\cos 2 \phi_{h}} \propto h_{1}^{q} \otimes H_{1}^{\perp, q \rightarrow h}+\frac{1}{Q}\left(f_{1}^{q} \otimes D_{1}^{q \rightarrow h}+\cdots\right)$ & $A_{U T}^{\sin \phi_{S}} \propto \frac{1}{Q}\left(h_{1}^{\perp, q} \otimes H_{1}^{\perp, q \rightarrow h}+f_{1 T}^{\perp, q} \otimes D_{1}^{q \rightarrow h}+\cdots\right)$ \\
$A_{U T}^{\sin \left(\phi_{h}-\phi_{S}\right)} \propto f_{1}^{\perp, q} \otimes D_{1}^{q \rightarrow h}+\cdots$ & $A_{U T}^{\sin \left(2 \phi_{h}-\phi_{S}\right)} \propto \frac{1}{Q}\left(h_{1}^{\perp, q} \otimes H_{1}^{\perp, q \rightarrow h}+f_{1 T}^{\perp, q} \otimes D_{1}^{q \rightarrow h}\right)$ \\
$A_{U T}^{\sin \left(\phi_{h}+\phi_{S}\right)} \propto h_{1}^{q} \otimes H_{1}^{\perp, q \rightarrow h}+\cdots$ & $A_{L T}^{\cos \phi_{S}} \propto \frac{1}{Q}\left(g_{1 T}^{q} \otimes D_{1}^{q \rightarrow h}+\cdots\right)$ \\
$A_{U T}^{\sin \left(3 \phi_{h}-\phi_{S}\right)} \propto h_{1 T}^{\perp, q} \otimes H_{1}^{\perp, q \rightarrow h}+\cdots$ & $A_{L T}^{\cos 3 \phi_{h}-\phi_{S}} \propto \frac{1}{Q}\left(g_{1 T}^{q} \otimes D_{1}^{q \rightarrow h}+\cdots\right)$ \\
\hline
\end{tabular}

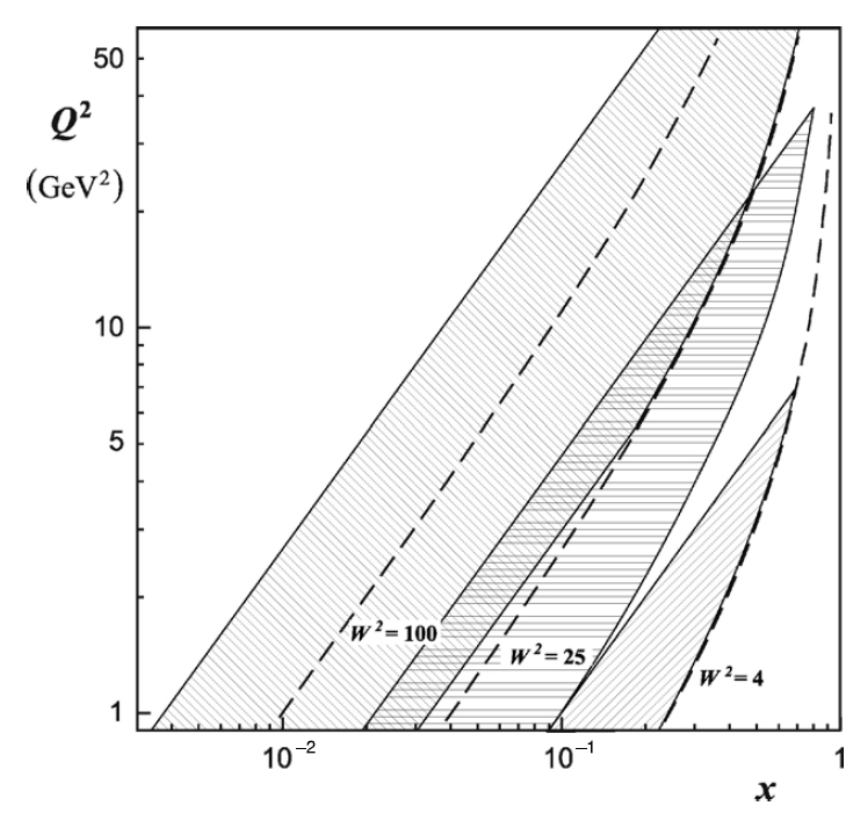

Figure 2. Phase space coverage of COMPASS, HERMES and JLab

transversity distribution, $h_{1}$ does not mix with gluons and evolves as a non-singlet density and at low $x, h_{1}$ is suppressed by the evolution with respect to $g_{1}$. This has important consequences for observables involving $h_{1}$ at low $x$ and large $Q^{2}$, such as the Drell-Yan double transverse asymmetry at collider energies.

(iv) The transversity distribution satisfies the following bound (Soffer [12]):

$$
\left|h_{1}^{q}(x)\right|<\frac{1}{2}\left|f_{1}^{q}(x)+g_{1}^{q}(x)\right|
$$

(v) The first moment of transversity give the tensor charge $\delta q$ by:

$$
\delta q=\int_{0}^{1} \mathrm{~d} x\left[h_{1}^{q}(x)-h_{1}^{\bar{q}}(x)\right]
$$

note that, due to charge-conjugation properties of the defining operator, the tensor charge is the first moment of a flavour non-singlet combination (quarks minus anti-quarks).
Today, the most direct information on transversity comes from SIDIS measurements with transversely polarised targets, which are complementary to the DY experiments and have the advantage of allowing a flavour separation by identification of the final state hadrons.

Among the various SIDIS observable related to transversity, the measurements performed so far have provided precise data on two of them: the Collins asymmetry [13] and the two-hadron asymmetry [14].

\subsection{The Collins asymmetry}

The main source of information on the transversity PDFs is at present the Collins asymmetry, which couples $h_{1}$ to the Collins fragmentation function $H_{1}^{\perp}$ :

$$
A_{U T}^{\sin \left(\phi_{h}+\phi_{S}\right)}(x) \simeq \frac{\sum_{q} e_{q}^{2} h_{1}^{q}(x) \otimes H_{1}^{\perp, q \rightarrow h}}{\sum_{q} e_{q}^{2} f_{1}^{q}(x) \otimes D_{1}^{q \rightarrow h}}
$$

where $\otimes$ indicate the convolution integrals between the quark transverse momentum and the transverse momentum acquired by the hadron in the fragmentation process with respect to the quark. In this case the asymmetry as a function of $x$ is obtained by integrating over the other kinematic variables such as $z, p_{\mathrm{T}}^{h}$ or $Q^{2}$.

The Collins asymmetry has been measured by COMPASS on deuterons $\left({ }^{6} \mathrm{LiD}\right)$ and on protons $\left(\mathrm{NH}_{3}\right)$ for unidentified hadrons (dominated by pions), $\pi$ and $K$ (fig. 3), by HERMES on protons, for $\pi$ and $K$ (fig. 4) and by Hall A on neutrons $\left({ }^{3} \mathrm{He}\right)$ for $\pi$ and $K$ (fig. 5 ).

Both in COMPASS and in HERMES (when the same sign convention is adopted ${ }^{1}$ ), the Collins asymmetries measured for charged $\pi$ on protons show a clear negative signal for $\pi^{+}$and a positive signal for $\pi^{-}$as a function of $x, z$ and $p_{\mathrm{T}}$. The asymmetries measured by COMPASS and HERMES have the same amplitude once the HERMES data are corrected for the depolarisation factor, giving important information on the kinematic dependencies of the transversity PDF and Collins FF. The trend is confirmed for $K^{+}$, while $K^{-}$and $K^{0}$ signals are smaller (or absent), and more statistics is needed. The null COMPASS results on deuteron allows to constrain $h_{1}^{d}$. A global analysis of COMPASS, HERMES and Belle data allowed first extractions of transversity PDFs and Collins FFs [15], showing that transversity function is sizeable and different

${ }^{1}$ The COMPASS convension is adopted for the following discussion. 

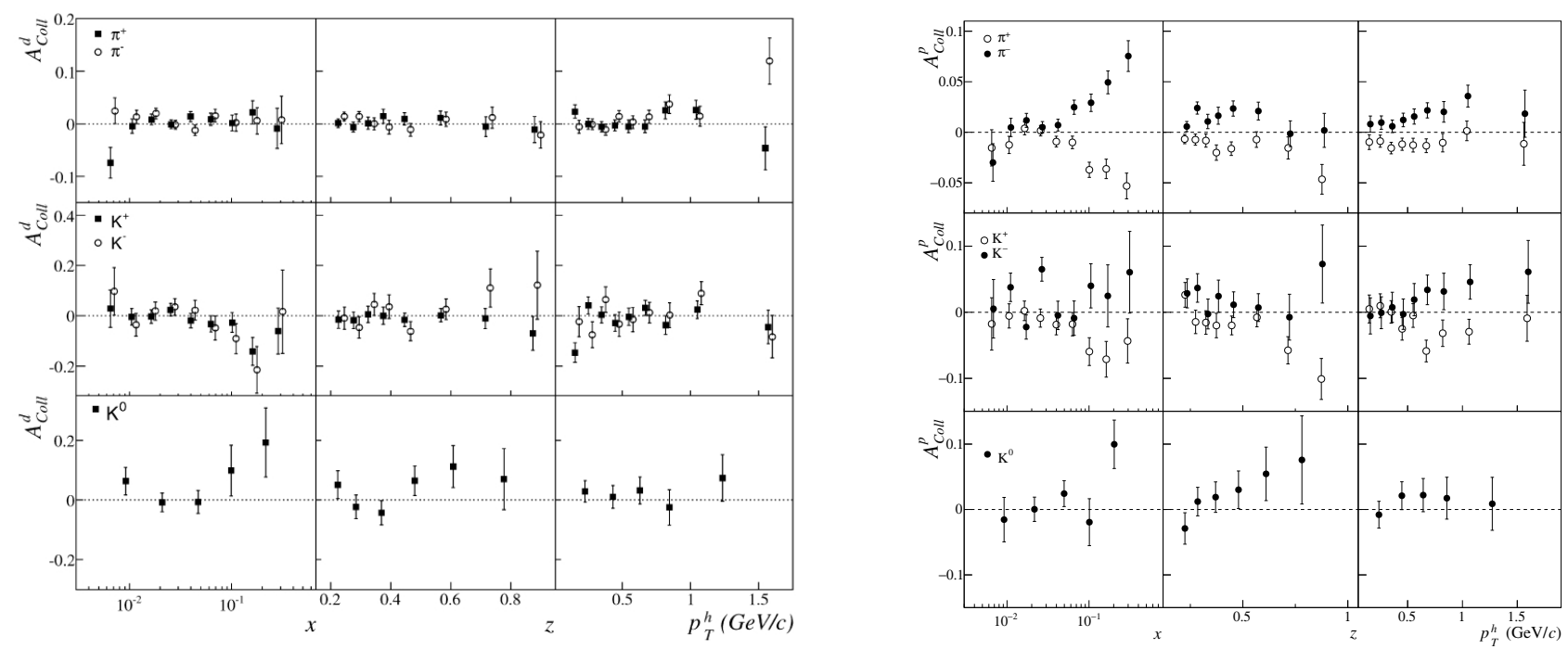

Figure 3. COMPASS measurements of the Collins asymmetries for $\pi^{ \pm}$and $K^{ \pm, 0}$ on deuterons (left) and protons(right).
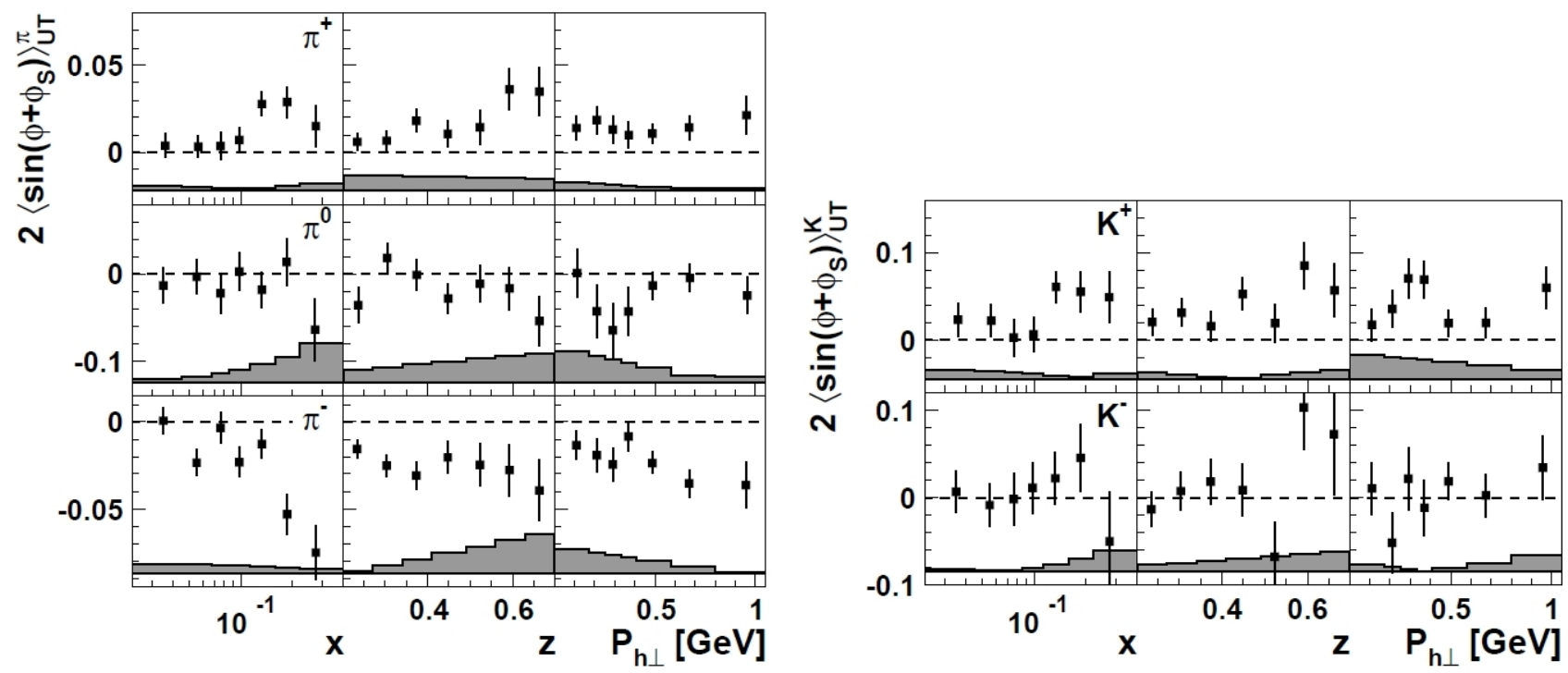

Figure 4. HERMES measurements of the Collins asymmetries on protons for $\pi^{ \pm}$(left) and $K^{ \pm}$(right).
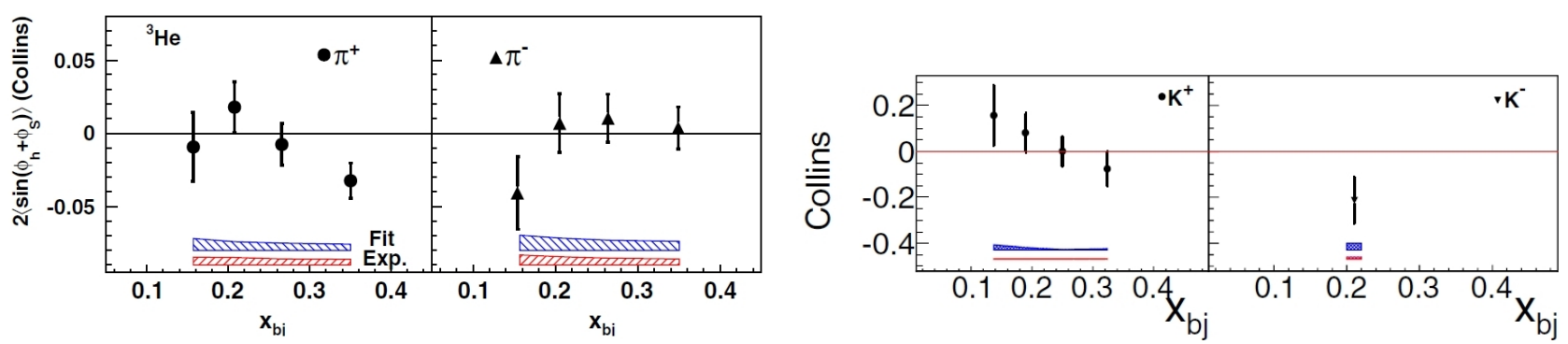

Figure 5. Hall A measurements of the Collins asymmetries on neutrons for $\pi^{ \pm}$(left) and $K^{ \pm}$(right). 
from zero, with opposite sign and similar amplitude for both the $u$ and $d$ quark, while at the same time favoured and unfavoured Collins FF are also large, with similar amplitude but opposite sign. The Collins asymmetries on $n$ from Hall A, are compatible with zero as expected from the fits of proton and deuteron data.

\subsection{The two-hadron asymmetry}

The transverse spin asymmetry in the distribution of the azimuthal plane of hadron pairs in the current jet of DIS have been measured by HERMES with the proton target and by COMPASS both with the deuteron and the proton targets. Also for this asymmetry there are some small differences between the analysis performed by the two experiments, that comprises a different definition of tranverse vector of the two hadrons (the azimuthal angle derived in the two cases eventually coincide in the $\gamma^{*} N$ system). Apart from slightly different DIS cuts, HERMES requires a missing mass larger than $2 \mathrm{GeV} / c^{2}$ to avoid contributions from exclusive two pion production. Also a minimum pion momentum of $1 \mathrm{GeV}$ is required for pion identification. In the COMPASS analysis, the event selection is more similar to that of the single hadron asymmetries, with a decreased minimum $z$ energy fraction required for both the hadrons $z_{1,2}>0.1$ and an upper limit of 0.9 . In the extraction of the asymmetries no dependence on the $\sin \theta$ component of the dihadron fragmentation function is taken into account since in the COMPASS kinematics the $\sin \theta$ distribution is strongly peaked at one $(\langle\sin \theta\rangle=0.94)$ and the $\cos \theta$ distribution is symmetric around zero. The larger $W$ accessible in COMPASS as compared to HERMES and JLab resulted in a higher sample of multi-hadron events and consequently more precise $2 h$ asymmetries. COMPASS results are shown in fig. 6 for both protons and deuteron and for different particles combinations. A clear signal in the valence region is only visible for $\pi^{+} \pi^{-}$combination.

Recently COMPASS pointed out that the dihadron asymmetry as functions of $x$ and the Collins asymmetries for both $\pi^{+}$and $\pi^{-}$show important similarities, i.e the dihadron asymmetry is slightly larger in magnitude, but very close to the values of the Collins asymmetry for positive hadrons and to the value of the negative pions, after reversing the sign of the asymmetry. Taking into account correlations between azimuthal angles of positive and negative pions in the two hadron sample, that suggests that in the multi-hadrons fragmentation of the struck quark azimuthal angles of positive and negative hadrons created in the event differ by $\approx \pi$, hinting for a common origin for the Collins mechanism and the dihadron fragmentation function, as originally suggested in the ${ }^{3} P_{0}$ Lund model [16], or the recursive string fragmentation model [17].

\section{The Sivers asymmetry}

The azimuthal asymmetry

$$
f_{q / N^{\uparrow}}\left(x, \vec{k}_{\perp}\right)-f_{q / N^{\uparrow}}\left(x,-\vec{k}_{\perp}\right)=2 \frac{\left(\hat{P} \times \vec{k}_{\perp}\right) \cdot \vec{S}}{M} f_{1 T}^{\perp, q}\left(x, k_{\perp}^{2}\right)
$$

constructed from the difference of the probability to find an unpolarised quark with transverse momentum $\vec{k}_{\perp}$ or $-\vec{k}_{\perp}$ inside a transversely polarised nucleon is proportional to the Sivers function $f_{1 T}^{\perp}$. If not null, such azimuthal asymmetry will therefore show that unpolarised quarks in a transversely polarised nucleon have a preferential motion direction (f.i. $f_{1 T}^{\perp}>0$ f.i. means that in a nucleon moving in the positive $\hat{z}$ direction and transversely polarised upwards in $\hat{y}$, the unpolarised quarks will tend to move to the right, towards $-\hat{x}$ ).

The Sivers functions are associated with the timereversal $(T)$ odd correlations $\left(\hat{P} \times \vec{k}_{\perp}\right) \cdot \vec{S}$, defining the Sivers function as a "T-odd distribution". It was shown that in order not to vanish this TMD PDF requires final state interactions in SIDIS, i.e. only gluon exchanges between the struck quark and the target remnant can generate a non-zero Sivers asymmetry. Since time reversal changes a future-pointing Wilson line into a past-pointing one, Tinvariance, rather than constraining $f_{1 T}^{\perp}$ to zero, gives a relation between processes that probe Wilson lines pointing in opposite time directions. In particular, since in SIDIS the Sivers asymmetry arises from the interaction between the spectator and the outgoing quark, whereas in Drell-Yan production it arises from the interaction between the spectator and an incoming quark, one gets a process dependent result in the measurement of the Sivers function:

$$
\left(f_{1 T}^{\perp}\right)_{\text {SIDIS }}=-\left(f_{1 T}^{\perp}\right)_{\mathrm{DY}}
$$

This relation is a direct consequence of the gauge structure of parton distribution functions, and its experimental check would be extremely important. The quark Sivers function has an exact gluonic counterpart, $f_{1}^{g \perp}$, which represents the distribution of unpolarised gluons in a transversely polarised hadron.

The main source of information on the Sivers PDFs is the measurement of the Sivers asymmetry in SIDIS, which couples $f_{1 T}^{\perp}$ to the unpolarised fragmentation function $D_{1}$ :

$$
A_{U T}^{\sin \left(\phi_{h}-\phi_{S}\right)}(x) \simeq \frac{\sum_{q} e_{q}^{2} f_{1 T}^{\perp, q}(x) \otimes D_{1}^{q \rightarrow h}}{\sum_{q} e_{q}^{2} f_{1}^{q}(x) \otimes D_{1}^{q \rightarrow h}}
$$

here again $\otimes$ indicates the convolution integrals. Again the asymmetry as a function of $x$ is obtained by integrating over the other kinematic variables such as $z, p_{\mathrm{T}}^{h}$ or $Q^{2}$.

As for the Collins asymmetry, the Sivers asymmetry has been measured by COMPASS on deuterons $\left({ }^{6} \mathrm{LiD}\right)$ and on protons $\left(\mathrm{NH}_{3}\right)$ for unidentified hadrons (dominated by pions), $\pi$ and $K$ (fig. 7), by HERMES on protons, for $\pi$ and $K$ (fig. 8 ) and by Hall A on neutrons $\left({ }^{3} \mathrm{He}\right.$ ) for $\pi$ (fig. 9).

For both COMPASS and HERMES the asymmetries for negative pions and kaons, as well as for neutral kaons are compatible with zero, while for positive pions and kaons there is a clear evidence for a positive signal. For COMPASS the signal extends over the full measured $x$ region and increases with $z$. As for HERMES, the $K^{+}$signal is larger than the $\pi^{+}$one, which indicates a possibly not negligible role of sea quarks. Unlike the case of the Collins asymmetry, the Sivers asymmetry measured by COMPASS at large $x$ for positive pions and kaons is smaller 

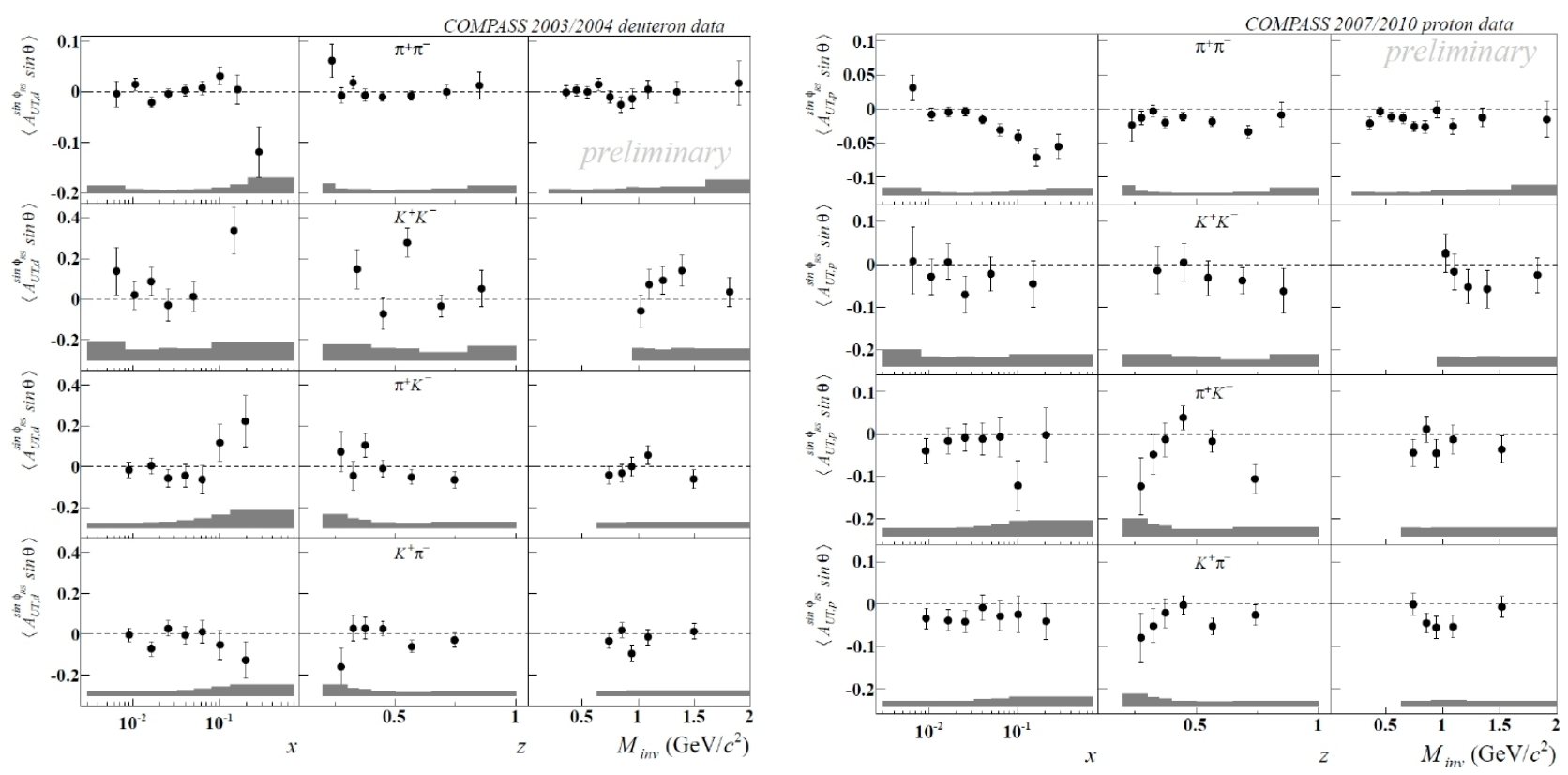

Figure 6. COMPASS measurements of the two identified hadron asymmetries of $\pi^{+} \pi^{-}, \pi^{+} K^{-}, K^{+} \pi^{-}$and $K^{+} K^{-}$on deuterons (left) and protons (right).
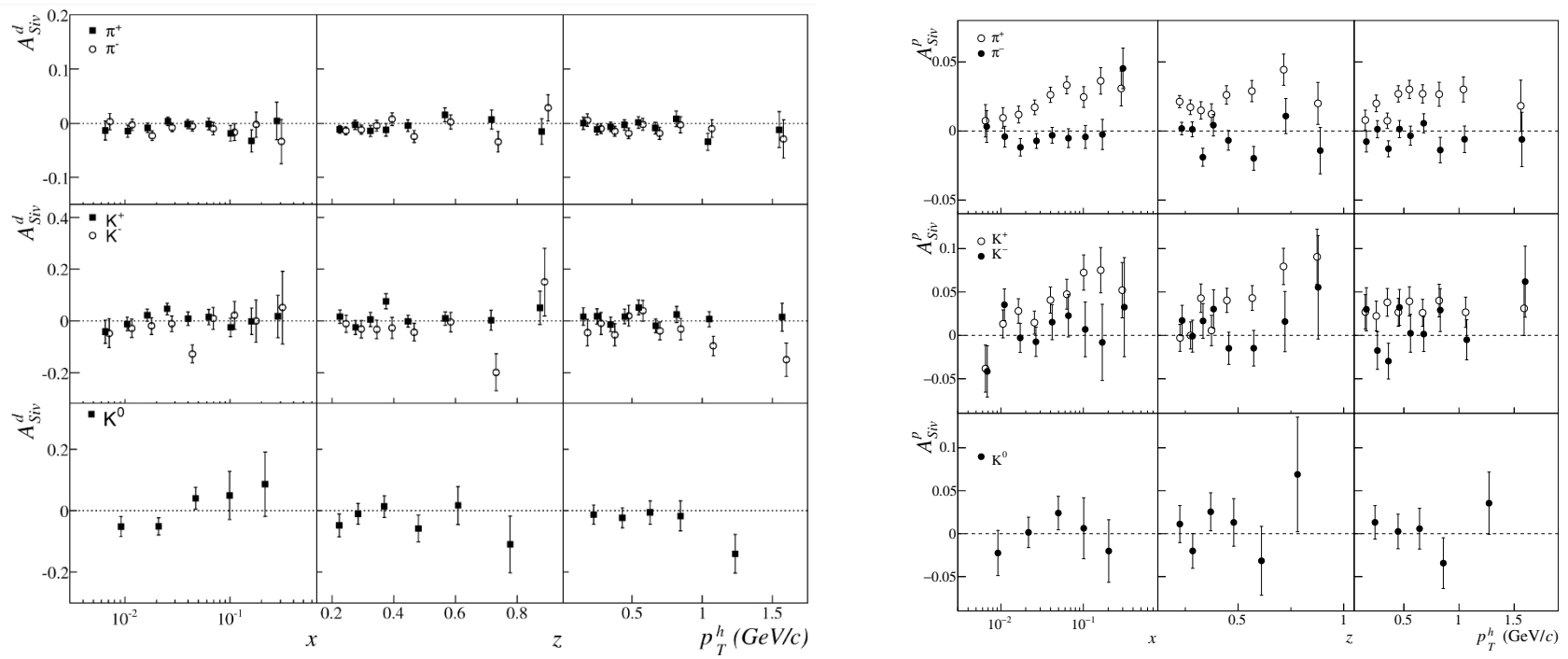

Figure 7. COMPASS measurements of the Sivers asymmetries for $\pi^{ \pm}$and $K^{ \pm, 0}$ on deuterons (left) and protons(right).

than the one from HERMES. Several fits, which include the recently revisited $Q^{2}$ evolution, were performed using HERMES asymmetries, COMPASS asymmetries on deuteron and for unidentified hadrons on proton, and JLab Hall A asymmetries on ${ }^{3} \mathrm{He}$. Some of these fits [18-20], which employ $Q^{2}$ TMD evolutions, are shown to well reproduce the results of the three experiments.

COMPASS has very recently measured the Sivers asymmetry for gluons [21] from the data collected with the ${ }^{6} \mathrm{LiD}$ transversely polarized target. The Sivers gluon asymmetry was extracted using a technique similar to the one adopted for the gluon helicity $\Delta g$ analysis [22, 23]. As expected from phenomenological studies of existing data $[24,25]$ the asymmetry is small (fig. 10) and com- patible with zero, within the present experimental precision. Higher precision is expected from the analysis of the transversely polarised COMPASS $\mathrm{NH}_{3}$ data.

\section{Multidimensional asymmetries: $x-Q^{2}$ binning}

The relative large statistics collected by COMPASS with a proton target allows to study, within a single $x$ bin, the behaviour of the asymmetries as a function of $Q^{2}$. This is particularly important also in view of the planned DrellYan measurement that will check the expected change of sign of the Sivers asymmetry, selecting events above $J / \psi$ 

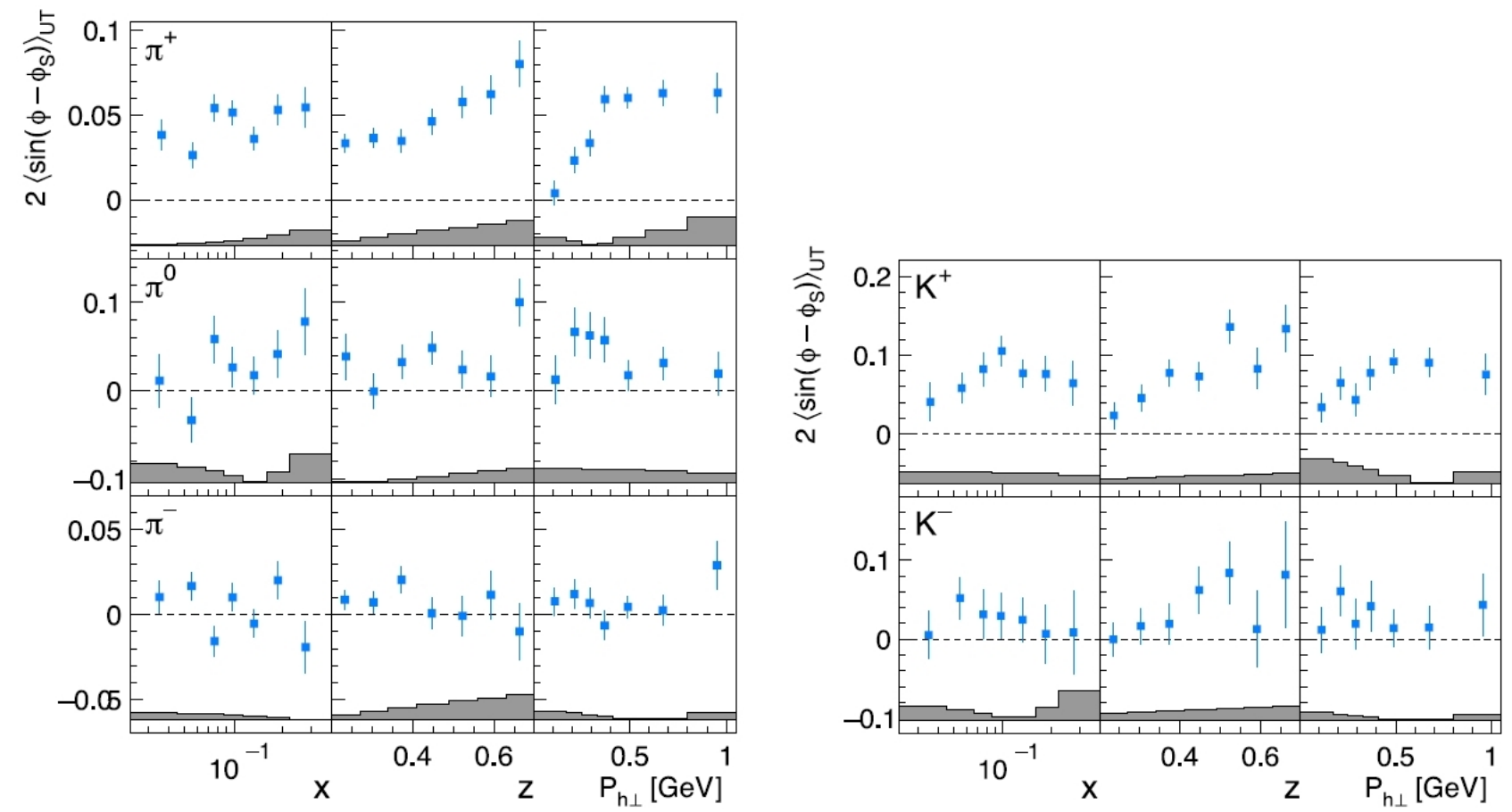

Figure 8. HERMES measurements of the Sivers asymmetries on protons for $\pi^{ \pm}$(left) and $K^{ \pm}$(right).
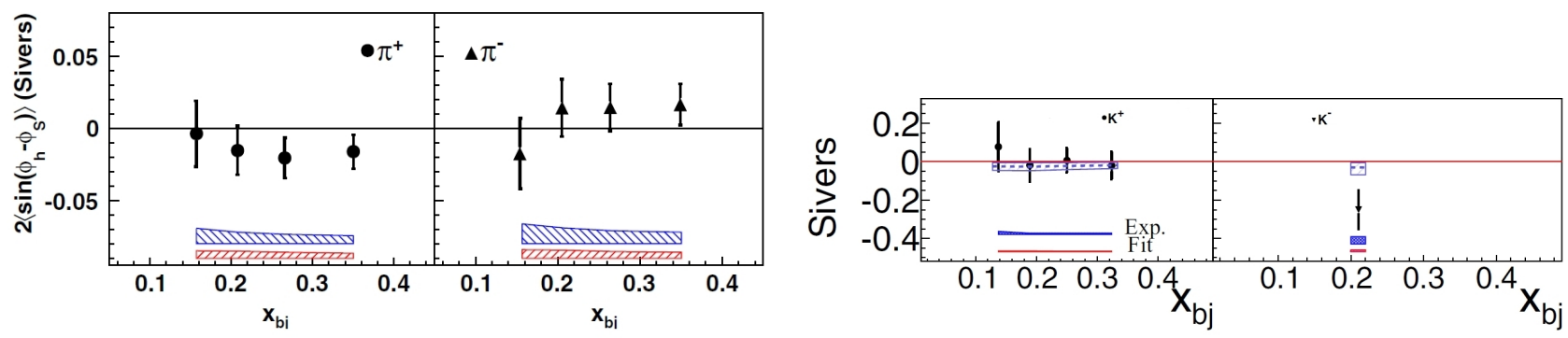

Figure 9. Hall A measurements of the Sivers asymmetries on neutrons for $\pi^{ \pm}$(left) and $K^{ \pm}$(right).

resonance, i.e. $Q^{2}>16(\mathrm{GeV} / c)^{2}$. Collins and Sivers asymmetries as a function of $x$ are shown in fig. 11

Non-zero effects were detected for asymmetries in all ranges. A clear signal is visible for Sivers with positive hadrons in all ranges and some hints of possible non-zero effect can be noticed for negative hadrons at relatively large $x-Q^{2}$. The Collins asymmetry is visible both for positive and negative hadrons in all $Q^{2}$-ranges except in the very low- $x$ region. A more completat multidimensional analysis is in preparation, aiming to present it at the forthcoming SPIN2014 Conference.

\section{Inclusive asymmetries}

Recently HERMES has studied single-spin asymmetries in inclusive electro-production of charged pions and kaons from transversely polarised protons [26]. These asymmetries were studied as a function of the azimuthal angle $\psi$ about the beam direction between the target-spin direction and the hadron production plane, the transverse hadron momentum $P_{T}$ relative to the direction of the incident beam, and the Feynman variable $x_{F}$. The $\sin \psi$ asymmetries are positive for $\pi^{+}$and $K^{+}$, slightly negative for $\pi^{-}$and consistent with zero for $K^{-}$. Measuring inclusive asymmetries with lepton beams allows (at variance to $A_{N}$ measurements with hadron beams) to investigate the different contribution from events with a lepton in the acceptance (DIS) and events without it (quasi-real production, or 'anti-tagged'). Figure 12 shows the asymmetries for the three subsamples (anti-tagged, DIS with $0.2<z<0.7$ and DIS with $z>0.7$ ) are shown. For the anti-tagged the asymmetries are almost identical to the full inclusive asymmetries, as expected by their much large statistics. Very large asymmetries are observed for the $z>0.7$ DIS sample (positive for $\pi^{+}$and negative for $\pi^{-}$) with values reaching up to 0.4 for the positive hadrons. The source of these large asymmetries is not fully understood, and is probably combinations of different processes, exclusive production of vector mesons, contributing up to $50 \%$ to the pions in the large $P_{T}$ region, on top of standard DIS. 

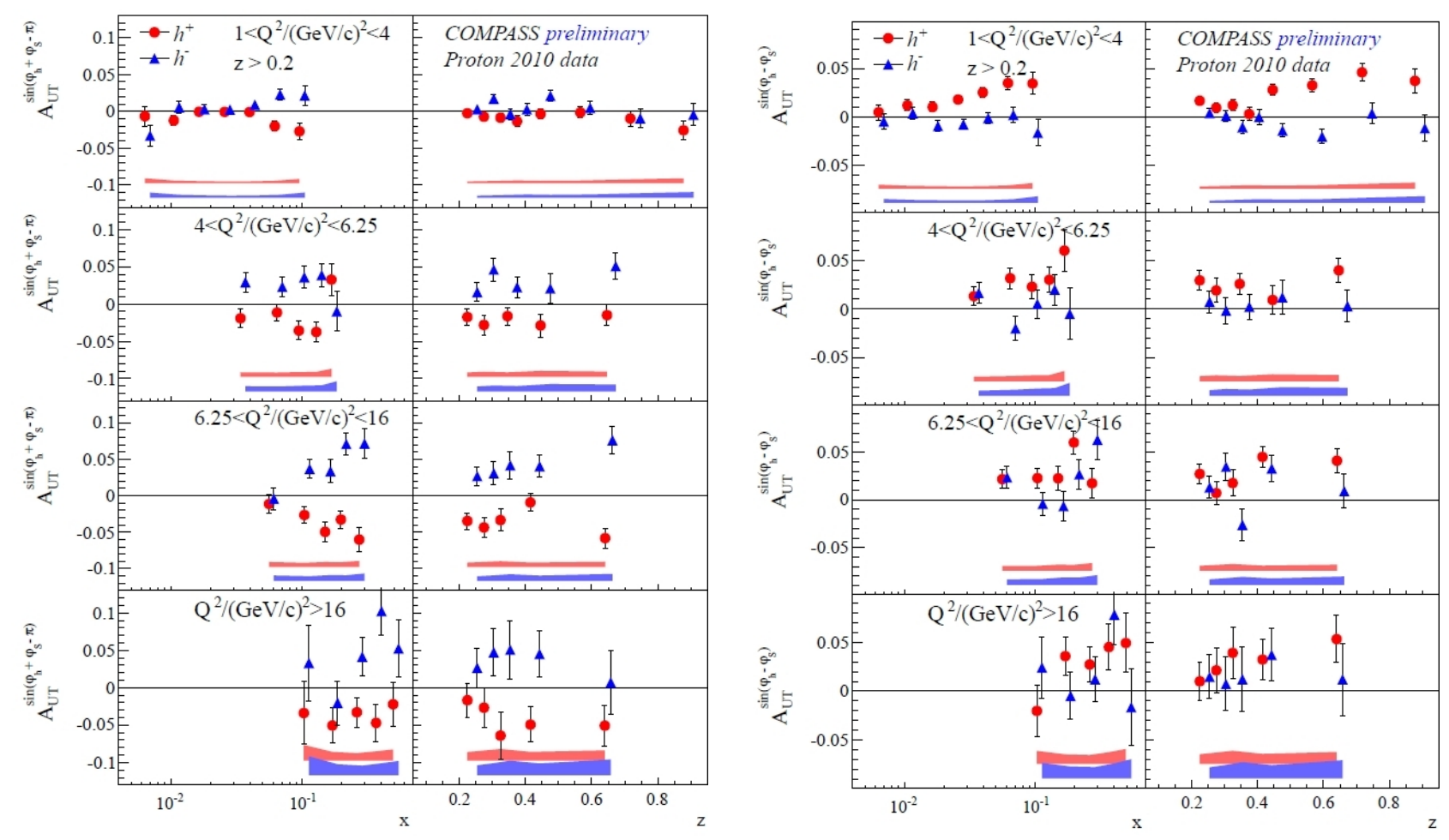

Figure 11. Collins (left) and Sivers (right) asymmetries for $4 Q^{2}$ bins as a function of $x$ and $z$.

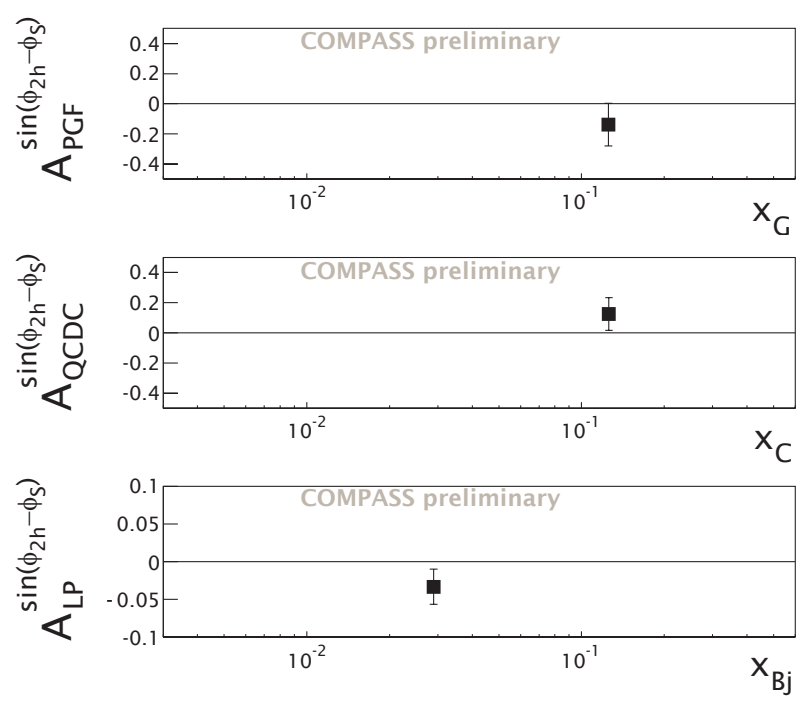

Figure 10. COMPASS Sivers asymmetry for gluons (top) together with the measured asymmetries for the QCD Compton process (middle) and the leading order $\gamma^{*} q \rightarrow q$ process (bottom).

\section{Other transverse momentum dependent single spin asymmetries}

Besides Collins and Sivers asymmetries, most of the other transverse spin asymmetries, containing the remaining six twist two TMD PDF are, at the present experimental precision, small or compatible with zero. An exception is the the only leading-twist LT-amplitude $A_{L T}^{\cos \left(\phi_{h}-\phi_{s}\right)}$ giv-

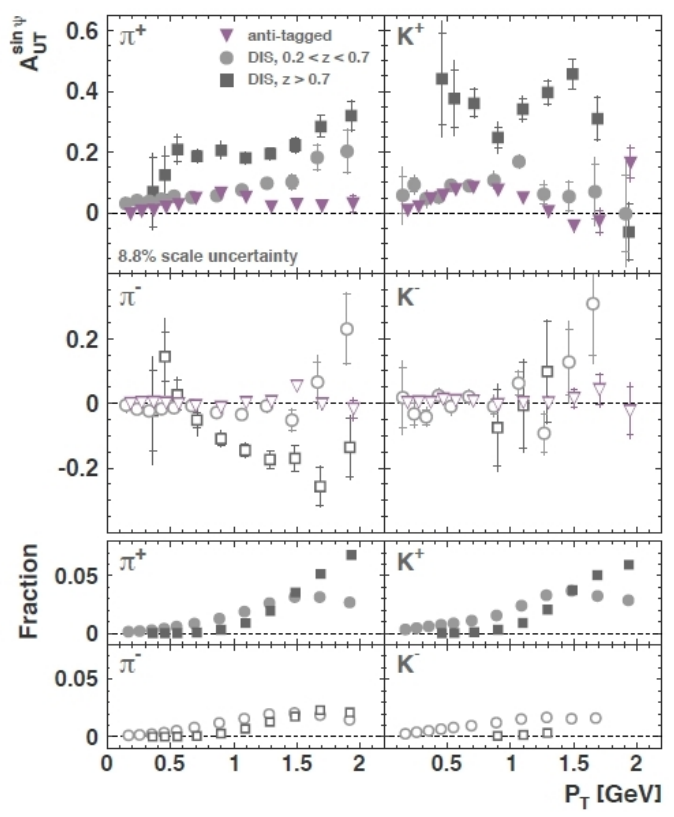

Figure 12. Hall A measurements of the Sivers asymmetries on neutrons for $\pi^{ \pm}$(left) and $K^{ \pm}$(right).

ing access to the worm-gear $g_{1 T}^{q}$ distribution function. $g_{1 T}^{q}\left(x, k_{T}^{2}\right)$ describes longitudinal polarisation of quarks in transversely polarised nucleon and is the only TMD that is both chiral-even and T-even and that does not involve finalstate interactions to exist. Similar to Collins and Sivers terms this asymmetry is expected to scale according to $\sim\left|\mathbf{P}_{h \perp}\right| . g_{1 T}^{q}\left(x, k_{T}^{2}\right)$ requires an interference between wave 


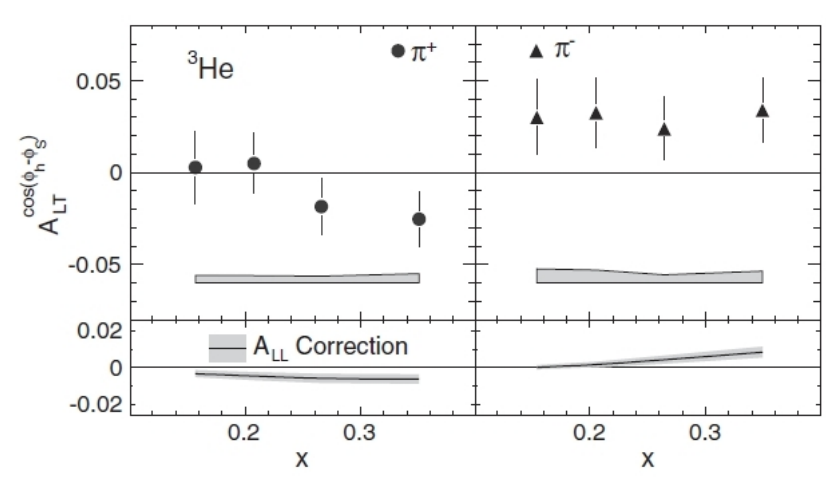

Figure 14. Hall A measurements of $A_{L T}^{\cos \left(\phi_{h}-\phi_{s}\right)}$ on neutrons showing some hint of non zero asimmetries, more evident for $\pi^{-}$.

function components differing by one unit of quark orbital angular momentum, therefore the observation of a nonzero result would provide direct evidence that quarks carry orbital angular momentum, constraining an important part of the nucleon spin sum rule [27].

COMPASS proton results for $A_{L T}^{\cos \left(\phi_{h}-\phi_{s}\right)}$ are presented in Fig. 13. The statistical uncertainties of all LTasymmetries are considerably larger than the ones of UT SSAs due to the relatively smaller $D(y)$ factors. Statistical precision achieved with proton data revealed a non-zero trend at relatively large $x$-region. JLab Hall A neutron results for $A_{L T}^{\cos \left(\phi_{h}-\phi_{s}\right)}$ are presented in Fig. 14. Also in this case there are hints of a non-zero trend at large $x$-region.

\section{Conclusions}

The study of the transverse spin and transverse momentum structure of hadrons has grown considerably in the last years and is now a central item of research of many laboratories around the world. In the last years, relevant experimental findings were:

- that there is a correlation between the spin of transversely polarised quarks and the $p_{\perp}$ of the hadrons created in the quark hadronisation process. Convincing evidence for this correlation has been provided by both SIDIS processes on transversely polarised nucleons and high energy $e^{+} e^{-}$annihilation into hadrons (this have made possible have a first measurement of the transversity distribution function);

- that there is also a non-zero correlation between the spin of a transversely polarised nucleon and the intrinsic transverse momentum of the quarks.

Still, many things remain to be done; the available SIDIS data cover only a limited phase space, and many more data are needed to obtain the $p_{\mathrm{T}}$ and $Q^{2}$ dependence of the asymmetries in the different $x$ and $z$ bins, that are needed to allow model independent extraction of the TMD functions.

In the near future more SIDIS data will be collected by JLab at $12 \mathrm{GeV}$, while COMPASS will perform the first ever polarised Drell-Yan process in $\pi^{-} p$, to check the change of sign of the Sivers function. COMPASS will also provide new measurements of azimuthal asymmetries in SIDIS on a liquid hydrogen target.

In an even more distant future, many projects have been proposed. The PAX experiment at FAIR aims to investigate Drell-Yan reactions in polarised antiprotonpolarised proton scattering (very clean way to address the transversity functions, but very challenging). Fixed target DY experiments scattering polarised protons on polarised protons are being planned at JPARC, in Japan, and at NICA, in Dubna. It is clear, however, that the future of this field strongly depends on the realization of the long time dreamed polarised electron-polarised proton collider. The US project is on his track as a joint BNL-JLab collaboration and has been approved as one of the 'Long range plans' of the NSAC, while recently, China is also studying the option to build a collider at the planned HIAF facility ('EIC@HIAF) with a lower energy/luminosity, $(\sqrt{s}$ similar to COMPASS) and both polarised electron and polarised proton beams.

\section{References}

[1] M. Alekseev, et al. (COMPASS Collaboration), Phys. Lett. B 673 (2009) 127.

[2] C. Adolph, et al. (COMPASS Collaboration), Phys. Lett. B 717 (2012) 376.

[3] C. Adolph, et al. (COMPASS Collaboration), Phys. Lett. B 717 (2012) 383.

[4] A. Airapetian, et al. (HERMES Collaboration), Phys. Lett. B693 (2010) 11.

[5] A. Airapetian, et al. (HERMES Collaboration), Phys. Rev. Lett. 103 (2009) 152002.

[6] X. Qian, et al. (The Jefferson Lab Hall A Collaboration), Phys. Rev. Lett. 107 (2011) 072003.

[7] Y.X. Zhao, et al. (The Jefferson Lab Hall A Collaboration), Phys. Rev. C 900, 055201 (2014).

[8] M. Diehl and S. Sapeta, Eur. Phys. J. C 41 (2005) 515.

[9] A. Bacchetta, M. Diehl, K. Goeke, A. Metz, P. Mulders and M. Schlegel, JHEP 0702, 093 (2007).

[10] X.-d. Ji, J.-p. Ma, F. Yuan, Phys. Rev. D71 (2005) 034005.

[11] X. Ji, J.-w. Qiu, W. Vogelsang, F. Yuan, Phys. Rev. D73 (2006) 094017.

[12] J. Soffer, Phys. Rev. Lett. 74 (1995) 1292.

[13] J.C. Collins, S.F. Heppelmann, G.A. Ladinsky, Nucl. Phys. B420 (1994) 565.

[14] A. Bacchetta, M. Radici, Phys. Rev. D67 (2003) 094002.

[15] M. Anselmino, et al., Nucl. Phys. Proc. Suppl. 191 (2009).

[16] B. Andersson and G. Gustafson and G. Ingelman and T. Sjöstrand Physics Reports 97 (1983) 31.

[17] X. Artru , arXiv: 1001.1061 [hep-ph].

[18] M. Anselmino, E. Boglione, S. Melis, Phys. Rev. D 86 (2012) 014028.

[19] P. Sun and F. Yuan, Phys. Rev. D 88 (2013) 114012. 


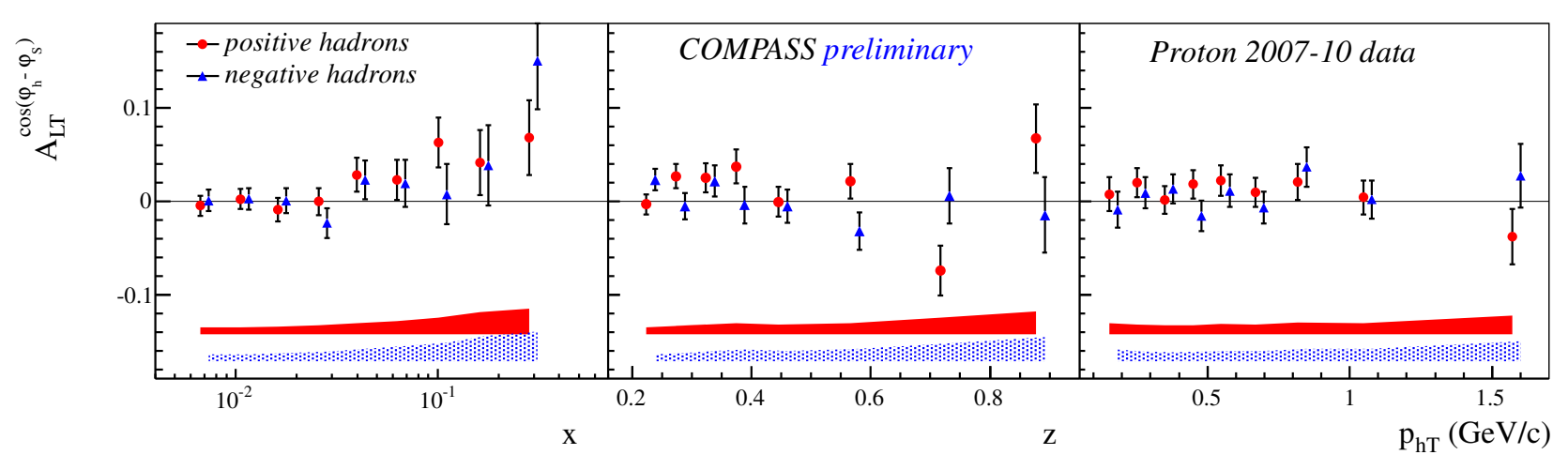

Figure 13. COMPASS measurement of $A_{L T}^{\cos \left(\phi_{h}-\phi_{s}\right)}$ on protons, showing some hint of non zero asimmetries.

[20] M.G. Echevarria et al., Phys. Rev. D 89 (2014) 074013.

[21] A. Szabelski for the COMPASS Collaboration, proceedings of Transversity 2014.

[22] C. Adolph et al. (COMPASS Collaboration), Phys. Lett. B 718 (2013) 922.

[23] C. Adolph et al. (COMPASS Collaboration), Phys. Rev. D 87 (2013) 052018.
[24] S. J. Brodsky and S. Gardner, Phys. Lett. B 643 (2006) 22.

[25] M. Anselmino, U. D’Alesio, S. Melis and F. Murgia, Phys. Rev. D 74 (2006) 094011.

[26] A. Airapetian, et al., (HERMES Collaboration), Phys. Lett. B 728 (2014) 183.

[27] R. Jaffe and A. Manohar, Nucl. Phys. B 337 (1990) 509. 